POS PROCEEDINGS

\title{
Excited State Effects in Nucleon Matrix Element Calculations
}

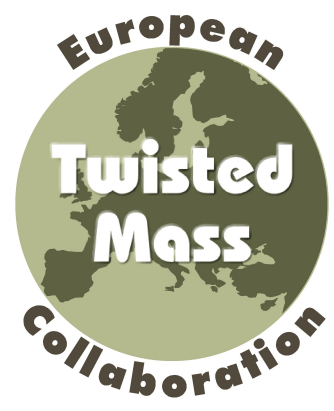

Constantia Alexandrou ${ }^{b, c}$, Martha Constantinou ${ }^{b}$, Simon Dinter ${ }^{* a}, \operatorname{Vincent} \operatorname{Drach}^{a}$, Karl Jansen ${ }^{a}$, Theodoros Leontiou ${ }^{d}$ and Dru B. Renner ${ }^{e}$

${ }^{a}$ NIC, DESY Zeuthen, Platanenallee 6, D-15738 Zeuthen, Germany

${ }^{b}$ Department of Physics, University of Cyprus, P.O. Box 20537, 1678 Nicosia, Cyprus

${ }^{c}$ Computation-based Science and Technology Research Center, The Cyprus Institute, 15

Kypranoros Str., 1645 Nicosia, Cyprus

${ }^{d}$ General Department, Frederick University, 1678 Nicosia, Cyprus

e Jefferson Lab, 12000 Jefferson Avenue, Newport News, VA 23606, USA

E-mail: simon.dinter@desy.de

\begin{abstract}
We perform a high-statistics precision calculation of nucleon matrix elements using an open sink method allowing us to explore a wide range of sink-source time separations. In this way the influence of excited states of nucleon matrix elements can be studied. As particular examples we present results for the nucleon axial charge $g_{A}$ and for the first moment of the isovector unpolarized parton distribution $\langle x\rangle_{u-d}$. In addition, we report on preliminary results using the generalized eigenvalue method for nucleon matrix elements. All calculations are performed using $N_{f}=2+1+1$ maximally twisted mass Wilson fermions.
\end{abstract}

XXIX International Symposium on Lattice Field Theory

July $10-162011$

Squaw Valley, Lake Tahoe, California

\footnotetext{
* Speaker.
} 


\section{Introduction}

Quantities related to nucleon structure are among the most challenging that can be computed within lattice QCD. During the past few years, there has been significant progress in these calculations: quark masses used nowadays are close to the physical ones, lattice spacings are small enough to allow for a controlled extrapolation to the continuum limit and volumes are sufficiently large to suppress finite volume effects. But still even for some simple observables like the nucleon axial charge $g_{A}$ or the average momentum of the unpolarized isovector parton distribution $\langle x\rangle_{u-d}$ the values obtained from lattice calculations differ from their corresponding experimental values. In Fig. 1 we show the relative deviation of lattice results for $\langle x\rangle_{u-d}$ from the value obtained in Ref. [1] (ABMK). We also show the deviation of results from different phenomenological extractions from deep inelastic scattering experiments as compared to that of ABMK. The deviations of the phenomenologically determined values for $\langle x\rangle_{u-d}$ between the different groups might be interpreted as a systematic error related to different data sets and different analysis/fitting strategies. However, as can be seen, the spread in the phenomenological number is much less than the relative deviation between lattice data and phenomenology. One possible reason for the discrepancy can be

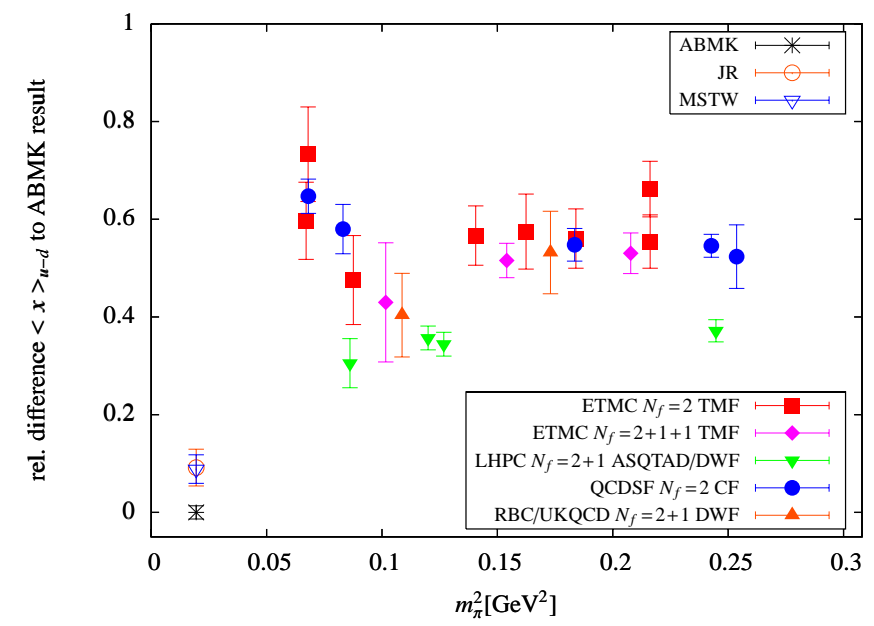

Figure 1: With the filled symbols we show the deviation of the values of $\langle x\rangle_{u-d}$ obtained from lattice calculations using twisted mass fermions (TMF) [2, 3] (ETMC), a hybrid action of domain wall fermions (DWF) on a stagerred sea [4] (LHPC), Clover fermions [5, 6] (QCDSF) and DWF [7] (RBC/UKQCD). The open symbols show the deviation of the phenomenological results [8] (JR) and [9] (MSTW) as compared to ABMK [1].

systematic effects that enter in the lattice calculations. Therefore the latter have to be thoroughly studied, which is a current important activity of many lattice collaborations. In these proceedings we focus on one potential systematic effect, namely excited state contributions. Furthermore we restrict ourselves to the two observables mentioned before, $g_{A}$ and $\langle x\rangle_{u-d}$, that are extracted from the matrix element at zero momentum transfer. These quantities are calculated in lattice QCD by studying the asymptotic behavior of suitable ratios of 3-point and 2-point correlation functions for large Euclidean time separations.

Several studies of $g_{A}$ and $\langle x\rangle_{u-d}$ at different values of the lattice spacing and various volumes $[2,7,4,5]$ indicate that for the pion masses considered, discretization and finite volume effects are likely not sufficient to explain the disagreement of the lattice calculations with the cur- 
rent experimental value of $g_{A}$ and the value of $\langle x\rangle_{u-d}$ from global analyses. Moreover, recent preliminary results [5] shows that the discrepancy persists even for pion masses almost as small as the physical one.

In order to clarify whether and to what extent excited state contributions affect the lattice calculation of matrix elements, we have performed a dedicated high-precision calculation of the 3-point functions for $g_{A}$ and $\langle x\rangle_{u-d}$. To be more precise, we have used roughly 7500 measurements for $g_{A}$ and about 23000 for $\langle x\rangle_{u-d}$, which enabled us to calculate the correlation functions needed for $g_{A}$ and $\langle x\rangle_{u-d}$ to sufficient accuracy even for large Euclidean time separations. This allowed us to study possible excited state contributions.

\section{Lattice techniques and details}

In this work, we have employed twisted mass Wilson fermions [10] at maximal twist. This lattice discretization has the advantage of an automatic $O(a)$-improvement, thus removing the necessity of additional operator specific improvements. We use gauge field configurations generated by the European Twisted Mass Collaboration (ETMC) with $N_{f}=2+1+1$ flavors and refer to Ref. [11] for further details of our lattice formulation.

To understand how the excited state contributions emerge from the calculation of nucleon matrix elements the main observation needed here is that to leading order we find two additional time dependent contributions for a matrix element $\langle N|\mathscr{O}| N\rangle$, namely

$$
\langle N|\mathscr{O}| N\rangle_{\text {lattice }}=\langle N|\mathscr{O}| N\rangle+A \exp \left(-\Delta M t^{\prime}\right)+B \exp \left[-\Delta M\left(t-t^{\prime}\right)\right] .
$$

Here $\Delta M$ is the mass gap between the nucleon ground state and the first excited state and $A$ and $B$ are constants depending on the particular choice of the lattice nucleon creation and annihilation operator, respectively. For details we refer the reader to Ref. [12]. For our precision calculation we restrict ourselves to only one ensemble with a pion mass of $m_{\pi} \approx 380 \mathrm{MeV}$ and a lattice spacing of $a \approx 0.078 \mathrm{fm}$. This pion mass is chosen sufficiently small in order to be relatively close to the physical pion mass on the one hand but still large enough to ensure that finite size effects can be safely neglected. Also, for such a pion mass, the calculations of the propagators do not require large computer resources, thus enabling us to collect the statistics necessary for the high precision we are aiming at. We expect discretization effects to be small due to the automatic $O(a)$-improvement, which is confirmed by previous calculations of nucleon matrix elements at three different lattice spacings smaller than $0.1 \mathrm{fm}[2,13,14]$ using $N_{f}=2$ maximally-twistedmass fermions. Let us point out in particular, that even though this analysis was performed using twisted-mass fermions, the important properties of excited state contributions are expected to be universal and independent of the particular lattice discretization used. Therefore we expect our conclusions to hold qualitatively also for different lattice actions.

For the precision calculation of the 3-point functions performed in this work we employed a method that is different from the standard sequential method, where the sink time slice is fixed. We fixed the time slice of the operator insertion as well as the operator to obtain a result for all sink time slices. This enables us to study the source-sink separation dependence of a particular matrix element. Note that in general the standard calculation is more desirable since it does not depend on the operator itself, whereas in the open sink method we have to restrict to a single operator. The interested reader is referred to Ref. [12] for a more detailed discussion. 
As stated in the introduction, we concentrate on two relatively simple but nonetheless phenomenologically relevant quantities. The first is the nucleon axial charge, $g_{A}$, playing an important role in the beta decay of the neutron and appearing as a low energy constant in effective chiral Lagrangians. It has been experimentally measured precisely and it is also straightforward to calculate in lattice QCD. However, the values obtained from various lattice calculations are typically $5 \%$ to $10 \%$ below the experimental result [15] while having themselves a statistical accuracy of the order of $1 \%$. The second observable is the lowest non-trivial moment of the unpolarized parton distribution function in isovector flavor combination, $\langle x\rangle_{u-d}$. It is determined phenomenologically from a global analysis of deep inelastic scattering data, and the discrepancy between the phenomenological and lattice values is even larger, about $50 \%$ to $60 \%$, as can be seen in Fig. 1. We would also like to stress that non-perturbative renormalization is employed for the bare matrix elements. The corresponding renormalization factors are calculated in the RI'MOM scheme and are matched to the $\overline{\mathrm{MS}}$ scheme at a scale of $(2 \mathrm{GeV})^{2}$. For more details we refer to Refs. [16, 3]. The values of the renormalization constants relevant here are $Z_{A}=0.774$ for the renormalization of the bare $g_{A}$ and $Z_{\langle x\rangle}=0.998$ for the renormalization of $\langle x\rangle_{u-d}[17]$.

\section{Results}

We performed an analysis of $g_{A}$ on a single $N_{f}=2+1+1$ ensemble characterized in Sec. 2 using the open sink method. The time slice of the operator insertion was fixed to $t^{\prime}=9 a$, chosen to safely suppress excited state contributions from the source, as can be verified from the 2-point function. We have used Gaussian smearing of the quark fields, including APE-smeared gauge links, in order to improve the overlap with the nucleon ground state. The result of the analysis using the open sink method is shown in Fig. 2 (left). In this figure we also compare the values obtained from the open sink analysis to the value of a standard analysis with a fixed source-sink separation of $12 a$ on the same ensemble. The value of $g_{A}$ does not show any dependence on the source-sink separation $t$ within statistical accuracy and thus demonstrates the absence of significant contribution from excited states. It is worth mentioning that, in order to reach a comparable statistical accuracy as the one obtained when using the fixed sink method with $t=12 a$ with 500 measurements, we had to perform roughly 7500 measurements when we take e.g. $t=18 a$. We have run the same analysis for $\langle x\rangle_{u-d}$. For the open sink method, we have chosen the operator insertion time to be $t^{\prime}=11 a$. We expect that for this choice excited state effects from the source are sufficiently suppressed. With a statistics of 23,000 measurements for $\langle x\rangle_{u-d}$ at a source-sink separation of $t=18 a$, we could equal the precision of the fixed sink method that was done with a source-sink separation of $t=12 a$ using 1300 measurements. In the right panel of Fig. 2 we plot $\langle x\rangle_{u-d}$ as a function of the source-sink separation $t$. We also indicate the value obtained from the fixed sink method analysis as well as the experimental result from a global analysis [1]. We observe that the values for $\langle x\rangle_{u-d}$ reach a plateau at larger values of the source-sink separation than what we have used in the fixed sink method and that the plateau value is shifted. Still, despite the fact that the results for larger values of $t$ decrease they clearly do not reach the phenomenological value. In order to estimate the residual dependence on $t$, we determined the value of $\langle x\rangle_{u-d}$ for an infinite source-sink separation by fitting the expected exponential behavior,

$$
\langle x\rangle_{u-d}+A \exp \left[-\Delta M\left(t-t^{\prime}\right)\right],
$$



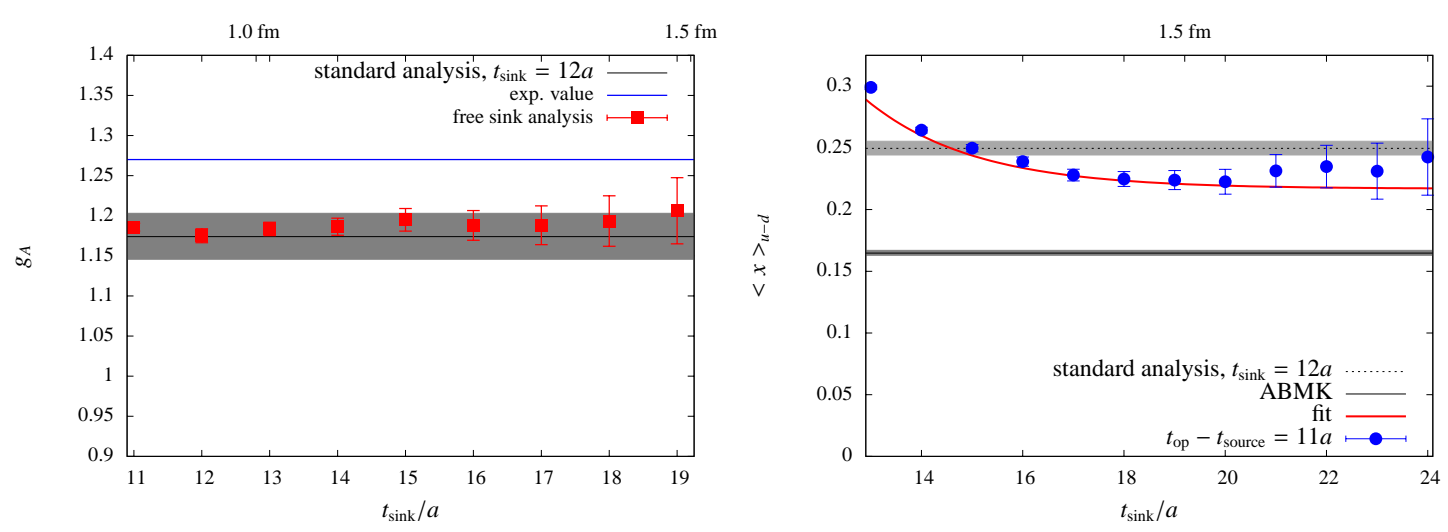

Figure 2: Left panel: Results for $g_{A}$ for a range of source-sink separations obtained from the open sink analysis on one $N_{f}=2+1+1$ ensemble. The light gray band indicates the result obtained from the fixed sink method using a source-sink separation of $12 a$ and the dark gray band shows the experimental value. Right panel: $\langle x\rangle_{u-d}$ for a range of source-sink separations obtained by means of the open sink method. The value (including errors) obtained from the fixed sink method using a source-sink separation of $12 a$ is indicated by the light gray band. The phenomenologically extracted value is shown with the dark gray band. The blue solid line corresponds to a fit described in the text.

to the lattice results with a fixed $t^{\prime}=11 a$. The result of this fit is $\langle x\rangle_{u-d}=0.22(1)$. This value is $12 \%$ lower than the result of $\langle x\rangle_{u-d}=0.250(6)$, obtained using $t=12 a$ in the fixed sink method. We estimated the error of the fit by varying the fit range and by comparing the results obtained by using a fixed parameter $\Delta M$ as well as by including $\Delta M$ in the fit. We have also
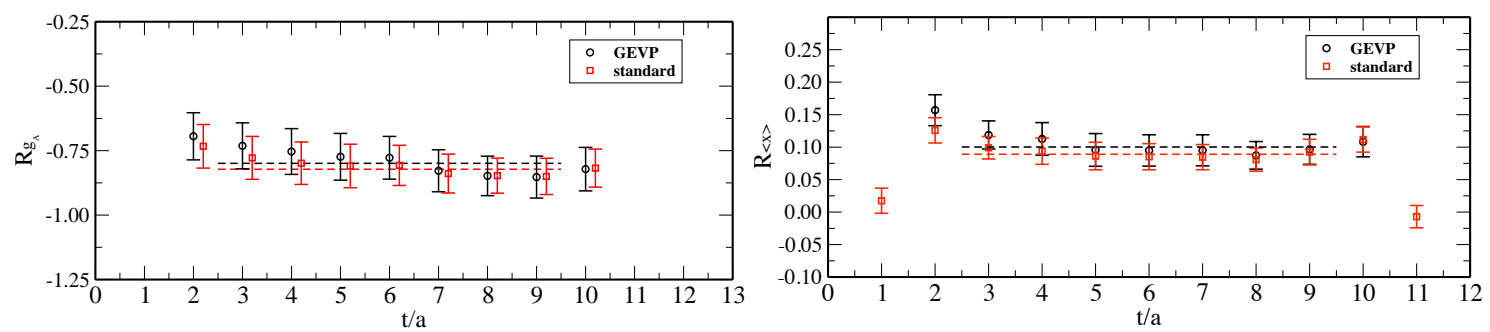

Figure 3: Ratio of 3- to 2-point functions for $g_{A}$ (left) and $\langle x\rangle_{u-d}$ (right) versus the time separation of the operator insertion from the source. The sink-source time separation $t_{\text {sink }}=12 a$. $N_{f}=2$ gauge configurations are used with pion mass $\sim 300 \mathrm{MeV}$ and $a=0.089 \mathrm{fm}$.

used a generalized eigenvalue (GEV) approach as first suggested in [18] and further developed and refined in Refs. $[19,20,21]$. We considered two variational basis: in the first, two interpolating fields are considered, namely the standard one $J_{N}(x)=\varepsilon^{a b c}\left(d^{T a}(x) C \gamma_{5} u^{b}(x)\right) u^{c}(x)$ and $J_{N}^{\prime}(x)=\varepsilon^{a b c}\left(d^{T a}(x) C u^{b}(x)\right) \gamma_{5} u^{c}(x)$. The latter is known to have small overlap with the nucleon state but a large one with the Roper. In Fig. 3 we compare the ratio of the three-point to the two-point function arising when using the GEV approach to the one using just the standard $J_{N}(x)$ interpolating field as we vary the time separation of the operator insertion form the source. As can be seen, for both $g_{A}$ and $\langle x\rangle_{u-d}$ the ratios are consistent and lead to the same value for these observables. In the second, three different levels of smearing are employed to calculated a $3 \times 3$ correlation matrix. In Fig. 4 we show the effective plateau matrix elements for fixed $t_{\mathrm{op}}=t_{\text {sink }} / 2$ and various $t_{0}$. From the preliminary results as shown in Fig. 4, we conclude that within the statis- 
tical errors we can achieve with our nucleon 3-point functions, the values obtained from the GEV are fully consistent with our standard method.

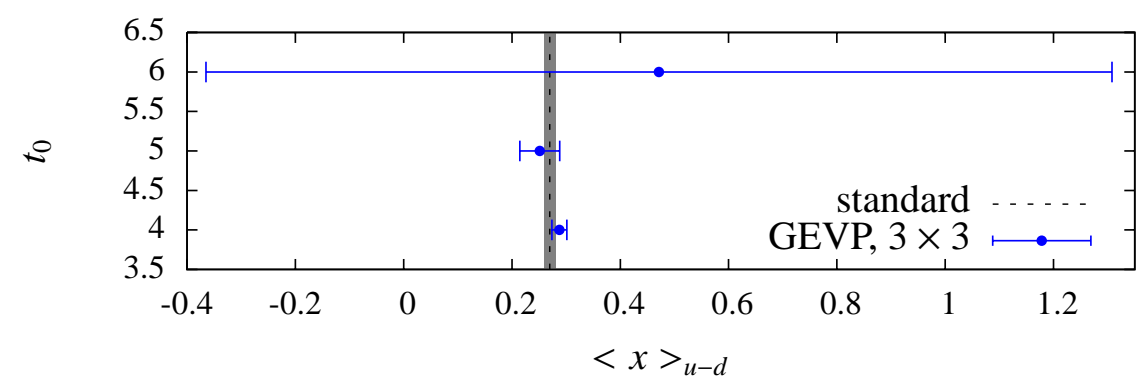

Figure 4: Comparison between the value obtained from a standard calculation of $\langle x\rangle_{\mathrm{u}-\mathrm{d}}$ using a fixed sourcesink separation of $12 a$ and preliminary results from a GEVP analysis using the same source-sink separation for a range of $t_{0}$.

\section{Summary and Conclusions}

We have performed precision calculations of $g_{A}$ and $\langle x\rangle_{u-d}$ for a single ensemble of gauge field configurations corresponding to a pion mass of about $380 \mathrm{MeV}$ with $N_{f}=2+1+1$ dynamical fermions employing a non-perturbative renormalization. We have studied the dependence of these quantities on the source-sink separation in order to assess the influence of excited states on the current lattice results for $g_{A}$ and $\langle x\rangle_{u-d}$. This is particularly important given that excited states may play a role in explaining the presently observed discrepancy between lattice computations and phenomenological evaluations of several important observables related to nucleon structure.

We find that for the here considered pion mass of about $380 \mathrm{MeV}$ and lattice spacing of $a \approx$ $0.078 \mathrm{fm}$, the contamination of excited states is negligible for $g_{A}$, but for $\langle x\rangle_{u-d}$ we observe an effect that is of the order of $10 \%$ compared to our previous calculations, in which the source-sink separation has been fixed to approximately $1 \mathrm{fm}$. This is an effect larger than the finite volume and lattice spacing effects found at this value of the pion mass, volume and lattice spacing. Moreover, this study shows that excited state contributions are operator dependent and should be investigated separately for each operator.

Motivated by a study where variational methods have been employed in the calculation of the $B^{*} B \pi$ coupling [21], we applied the generalized eigenvalue method [18, 19] to calculate these quantities. The results of this analysis were consistent with the standard method. This indicates that in order to extract excited state effects, for which the GEVP method is expected to be very efficient, a significantly higher accuracy of the lattice data are needed.

\section{Acknowledgments}

This work is coauthored in part by Jefferson Science Associates, LLC under U.S. DOE Contract No. DE-AC05-06OR23177. It was partly supported by the Cyprus Research Promotion

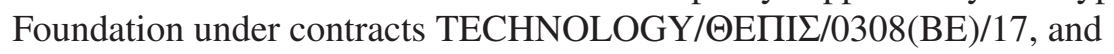
$\triangle$ IAKPATIKE $2 / \mathrm{KY}$-ГA/0310/02 and by the Research Executive Agency of the European Union under Grant Agreement number PITN-GA-2009-238353 (ITN STRONGnet). It is additionally supported in part by the DFG Sonderforschungsbereich Transregio SFB/TR9. Numerical calculations have been performed using HPC resources provided by Jülich Supercomputing Centre at the research center in Jülich. 


\section{References}

[1] S. Alekhin, J. Blumlein, S. Klein, and S. Moch. The 3, 4, and 5-flavor NNLO Parton from Deep-Inelastic-Scattering Data and at Hadron Colliders. Phys.Rev., D81:014032, 2010.

[2] C. Alexandrou, J. Carbonell, M. Constantinou, P.A. Harraud, P. Guichon, et al. Moments of nucleon generalized parton distributions from lattice QCD. Phys.Rev., D83:114513, 2011.

[3] S. Dinter, C. Alexandrou, M. Constantinou, V. Drach, K. Jansen, and D. Renner. Nucleon matrix elements with $N_{f}=2+1+1$ maximally twisted fermions. PoS, LATTICE2010:135, 2010.

[4] J.D. Bratt et al. Nucleon structure from mixed action calculations using $2+1$ flavors of asqtad sea and domain wall valence fermions. Phys.Rev., D82:094502, 2010.

[5] D. Pleiter et al. Nucleon form factors and structure functions from $\mathrm{N}(\mathrm{f})=2$ Clover fermions. $P o S$, LATTICE2010:153, 2010.

[6] A. Sternbeck. Private communication for QCDSF, 2011.

[7] Y. Aoki, T. Blum, H.-W. Lin, S. Ohta, S. Sasaki, et al. Nucleon isovector structure functions in (2+1)-flavor QCD with domain wall fermions. Phys.Rev., D82:014501, 2010.

[8] P. Jimenez-Delgado and E. Reya. Dynamical NNLO parton distributions. Phys.Rev., D79:074023, 2009.

[9] A.D. Martin, W.J. Stirling, R.S. Thorne, and G. Watt. Uncertainties on alpha(S) in global PDF analyses and implications for predicted hadronic cross sections. Eur.Phys.J., C64:653-680, 2009.

[10] R. Frezzotti and G.C. Rossi. Chirally improving Wilson fermions. 1. O(a) improvement. JHEP, 0408:007, 2004.

[11] R. Baron, Ph. Boucaud, J. Carbonell, A. Deuzeman, V. Drach, et al. Light hadrons from lattice QCD with light (u,d), strange and charm dynamical quarks. JHEP, 1006:111, 2010.

[12] S. Dinter, C. Alexandrou, M. Constantinou, V. Drach, K. Jansen, and D.B. Renner. Precision Study of Excited State Effects in Nucleon Matrix Elements. Phys.Lett., B704:89-93, 2011.

[13] C. Alexandrou et al. Axial Nucleon form factors from lattice QCD. Phys.Rev., D83:045010, 2011.

[14] C. Alexandrou. Hadron Structure and Form Factors. PoS, LATTICE2010:001, 2010.

[15] K. Nakamura et al. Review of particle physics. J.Phys.G, G37:075021, 2010.

[16] C. Alexandrou, M. Constantinou, T. Korzec, H. Panagopoulos, and F. Stylianou. Renormalization constants for 2-twist operators in twisted mass QCD. Phys.Rev., D83:014503, 2011.

[17] C. Alexandrou, M. Constantinou, T. Korzec, H. Panagopoulos, and F. Stylianou. Renormalization constants for one-derivative fermion operators in twisted mass QCD. PoS, LATTICE2010:224, 2010.

[18] Martin Luscher and Ulli Wolff. How to calculate the elastic scattering matrix in two-dimensional quantum field theories by numerical simulation. Nucl.Phys., B339:222-252, 1990.

[19] B. Blossier, M. Della Morte, G. von Hippel, T. Mendes, and R. Sommer. On the generalized eigenvalue method for energies and matrix elements in lattice field theory. JHEP, 0904:094, 2009.

[20] B. Blossier, M. Della Morte, N. Garron, G. von Hippel, T. Mendes, H. Simma, and R. Sommer. HQET at order 1/m: III. Decay constants in the quenched approximation. JHEP, 1012:039, 2010.

[21] J. Bulava, M. Donnellan, and R. Sommer. On the computation of hadron-to-hadron transition matrix elements in lattice QCD. 2011. 